\title{
High photoresponsivity in a GaAs film synthesized on glass using a pseudo-single- crystal Ge seed layer
}

Cite as: Appl. Phys. Lett. 114, 142103 (2019); https://doi.org/10.1063/1.5091714

Submitted: 04 February 2019 . Accepted: 26 March 2019 . Published Online: 11 April 2019

T. Nishida, K. Moto (D, N. Saitoh, N. Yoshizawa, T. Suemasu, and K. Toko
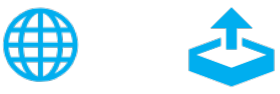

\section{ARTICLES YOU MAY BE INTERESTED IN}

Effect of Ge doping on growth stress and conductivity in $\mathrm{Al}_{\mathrm{X}} \mathrm{Ga}_{1-\mathrm{X}} \mathrm{N}$

Applied Physics Letters 114, 142101 (2019); https://doi.org/10.1063/1.5080680

Light-harvesting for high quantum efficiency in InAs-based InAs/GaAsSb type-II superlattices long wavelength infrared photodetectors

Applied Physics Letters 114, 141102 (2019); https://doi.org/10.1063/1.5086792

Anisotropic phonon properties and effective electron mass in a- $\mathrm{Ga}_{2} \mathrm{O}_{3}$

Applied Physics Letters 114, 142102 (2019); https://doi.org/10.1063/1.5086731

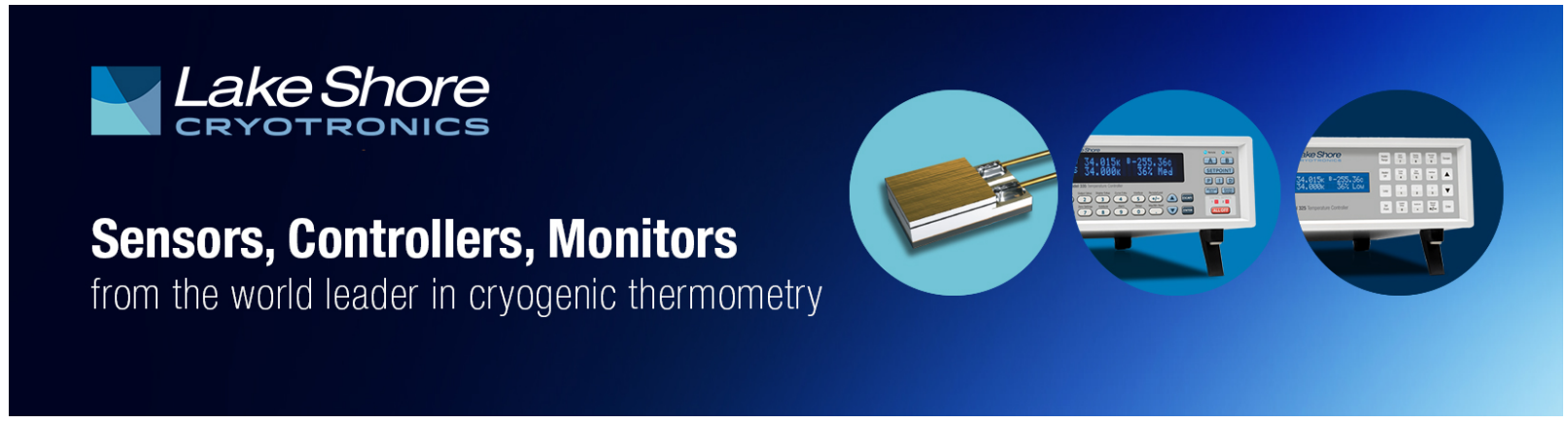




\title{
High photoresponsivity in a GaAs film synthesized on glass using a pseudo-single-crystal Ge seed layer
}

\author{
Cite as: Appl. Phys. Lett. 114, 142103 (2019); doi: 10.1063/1.5091714 \\ Submitted: 4 February 2019 • Accepted: 26 March 2019 • \\ Published Online: 11 April 2019
}

T. Nishida, ${ }^{7}$ K. Moto, ${ }^{7}$ (D) N. Saitoh, ${ }^{2}$ N. Yoshizawa, ${ }^{2}$ T. Suemasu, ${ }^{1}$ and K. Toko ${ }^{1, a)}$

\begin{abstract}
AFFILIATIONS
${ }^{1}$ Institute of Applied Physics, University of Tsukuba, 1-1-1 Tennodai, Tsukuba, Ibaraki 305-8573, Japan

${ }^{2}$ Electron Microscope Facility, TIA, AIST, 16-1 Onogawa, Tsukuba 305-8569, Japan
\end{abstract}

${ }^{a)}$ Author to whom correspondence should be addressed: toko@bk.tsukuba.ac.jp.

\begin{abstract}
Research to synthesize a high-quality GaAs film on an inexpensive substrate has been continuing for decades in the quest to develop a solar cell that achieves both high efficiency and low-cost. Here, we applied a large-grained Ge layer on glass, formed by Al-induced layer exchange, to an epitaxial template for a GaAs film. The GaAs film, grown epitaxially from the Ge seed layer at $520^{\circ} \mathrm{C}$, became a pseudosingle crystal (grain size $>100 \mu \mathrm{m}$ ) with high (111) orientation. Reflecting the large grain size, the internal quantum efficiency reached $70 \%$ under a bias voltage of $1.0 \mathrm{~V}$. This value approaches that of a simultaneously formed GaAs film on a single-crystal Ge wafer and is the highest for a GaAs film synthesized on glass at a low temperature. The application of a Ge seed layer formed by layer exchange offers excellent potential to develop high-efficiency thin-film solar cells with III-V compound semiconductors based on low-cost glass substrates.
\end{abstract}

Published under license by AIP Publishing. https://doi.org/10.1063/1.5091714

The highest conversion efficiency of solar cells has been updated with III-V compound semiconductors. ${ }^{1-3}$ However, since these solar cells use expensive single-crystal Ge or GaAs-based wafers, their applications have been limited to space use. If those substrates can be replaced with an inexpensive substrate, such as glass or a flexible substrate, III-V compound semiconductor solar cells will be the best candidate for the next generation of solar cells used for area-limited devices requiring a large amount of power, such as vehicles and mobile terminals. ${ }^{4,5}$ Although such solar cells have been achieved using transfer techniques so far, ${ }^{6-8}$ further cost reduction is still necessary for consumer applications.

Conversely, the direct synthesis of polycrystalline GaAs thin films on low-cost substrates has also been vigorously studied. The conversion efficiency of solar cells based on polycrystalline GaAs strongly depends on the grain size because the grain boundaries act as recombination centers. ${ }^{9,10}$ In a polycrystalline solar cell, if the grain size is sufficiently larger than the thickness of the light-absorbing layer, the conversion efficiency shows a value approaching that of a single crystal solar cell. Such a polycrystalline thin film can be called a pseudosingle crystal. So far, researchers have been investigating various techniques including vacuum depositon, ${ }^{11-17}$ crystallization of amorphous films, ${ }^{18-20}$ and chemical synthesis. ${ }^{21,22}$ However, it is still difficult to synthesize a large-area GaAs thin film with large grains $(>1 \mu \mathrm{m})$ on inexpensive amorphous substrates. In line with this, research to form a large-grained Ge thin film on an inexpensive substrate and epitaxially grow GaAs has been investigated for decades because Ge is lattice matched to GaAs. ${ }^{23,24}$ Dutta et al. sequentially grew Ge and GaAs films on a biaxially textured oxide layer formed on metal tape and demonstrated a conversion efficiency of $11.5 \%,{ }^{25}$ which is currently the highest efficiency for a low-cost GaAs thin-film solar cell. Because the grain size of the GaAs film is still small (a few micrometers), its enlargement will further improve the efficiency.

Metal-induced layer exchange is known as a method for forming a large-grained Ge thin film at low temperature on inexpensive substrates such as glass ${ }^{26-32}$ and even plastic. ${ }^{33-35}$ We achieved highly (111)-oriented Ge thin films with large grains $(>100 \mu \mathrm{m})$ using Alinduced layer exchange (ALILE) ${ }^{28,32}$ Because the light absorption thickness for GaAs is approximately $3.5 \mu \mathrm{m}$ owing to its large absorption coefficient, GaAs grown epitaxially on the ALILE-Ge layer will behave as a pseudosingle crystal. In this study, a pseudosingle crystal GaAs thin film is formed below the heat proof temperature of glass $\left(<550^{\circ} \mathrm{C}\right)$ using ALILE-Ge on glass as a seed layer. The resulting GaAs film exhibits a photoresponsivity comparable to a single-crystal GaAs film grown on a Ge wafer. 
Figure 1(a) shows the sample preparation procedure. A $50 \mathrm{~nm}$ thick Ge seed layer was prepared on a glass substrate using ALILE. ${ }^{28,32}$ In the ALILE process, $50 \mathrm{~nm}$-thick $\mathrm{Al}$ and $70 \mathrm{~nm}$-thick amorphous $\mathrm{Ge}$ (a-Ge) thin films were sequentially prepared on $\mathrm{a} \mathrm{SiO}_{2}$ glass substrate at room temperature using RF magnetron sputtering (base pressure: $3 \times 10^{-4} \mathrm{~Pa}$ ) with Ar plasma. A diffusion-controlling $\mathrm{AlO}_{x}$ layer was prepared between the $\mathrm{Al}$ and $\mathrm{a}-\mathrm{Ge}$ layers by air exposure ( $5 \mathrm{~min}$ ). The sample was annealed at $350^{\circ} \mathrm{C}$ for $50 \mathrm{~h}$ in $\mathrm{N}_{2}$. Although the annealing time is too long for the manufacturing process, it can be shortened by initially doping $\mathrm{Ge}$ in $\mathrm{Al}$ and by facilitating $\mathrm{Ge}$ diffusion into $\mathrm{Al}$ (e.g., modulating the $\mathrm{Ge} / \mathrm{Al}$ interlayer). ${ }^{30}$ After annealing, the sample was treated in an $\mathrm{H}_{2} \mathrm{O}_{2}$ (50\%) solution followed by an HF solution (1.5\%) to remove "Ge islands" and the $\mathrm{Al}$ layer and obtain a good Ge surface. $^{32}$ The resulting ALILE-Ge seed layer was highly (111)-oriented and large-grained $(>100 \mu \mathrm{m})$ as shown in the electron backscatter diffraction (EBSD) images in Figs. 1(b) and 1(c). The (001) orientation control of Ge is also possible using ALILE, while it reduces the grain size of Ge to approximately $10 \mu \mathrm{m} .{ }^{31}$ Some previous studies reported that $\mathrm{Ge}(111)$, especially with a few degrees of angle, is effective in suppressing antiphase domains in GaAs. ${ }^{36}$ Figure 1(d) shows that the ALILE-Ge is fortunately self-organized in the off angle (111) plane. Figure $1(\mathrm{e})$ shows that the ALILE-Ge consists mainly of high-angle grain boundaries because the grains are randomly oriented in the inplain direction. A GaAs layer was formed by molecular beam epitaxy (MBE, base pressure: $5 \times 10^{-8} \mathrm{~Pa}$ ) where $\mathrm{Ga}$ and As atoms were supplied by Knudsen cells with a GaAs deposition rate of $200 \mathrm{~nm} / \mathrm{h}$. Because this study focused on the demonstration of the potential of ALILE-Ge as a seed layer of GaAs, we used the MBE system and set the GaAs thickness to $500 \mathrm{~nm}$. The seeding technique will be applicable to thicker GaAs and mass production techniques such as chemical

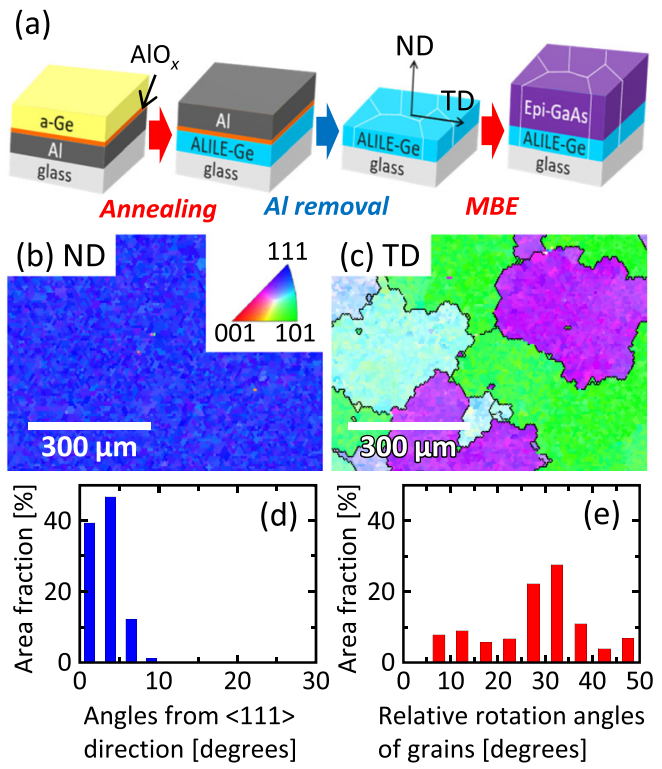

FIG. 1. (a) Schematic of the sample preparation. EBSD images of the ALILE-Ge seed layer in the (b) normal direction (ND) and (c) transverse direction (TD). The coloration indicates the crystal orientation, refer to the legend in the inset. The black solid lines in (c) indicate random grain boundaries. Distribution histograms of the (d) angles from the $\langle 111\rangle$ direction and (e) relative rotation angles of the grains. vapor deposition. The GaAs layers were prepared at a growth temperature, $T_{\mathrm{g}}$, ranging from 200 to $520^{\circ} \mathrm{C}$. For comparison, GaAs layers were formed on a single-crystal p-type $\mathrm{Ge}(111)$ substrate (c-Ge, resistivity $<10^{-2} \Omega \mathrm{cm}$ ) and a bare $\mathrm{SiO}_{2}$ glass substrate. For the photoresponse measurement, ITO electrodes ( $1 \mathrm{~mm}$ in diameter) were prepared on the GaAs surface using RF magnetron sputtering at room temperature.

Raman spectroscopy (spot diameter: $1 \mu \mathrm{m}$ and wavelength: $532 \mathrm{~nm}$ ) was used to determine the crystal state of the GaAs films. Figure 2(a) shows that the samples with ALILE-Ge for $T_{\mathrm{g}} \geq 370^{\circ} \mathrm{C}$ exhibit sharp peaks corresponding to the transverse optical mode $\left(\sim 270 \mathrm{~cm}^{-1}\right)$ and the longitudinal optical mode $\left(\sim 290 \mathrm{~cm}^{-1}\right)$ of crystalline GaAs, while that for $T_{\mathrm{g}}=200^{\circ} \mathrm{C}$ exhibits broad peaks corresponding to amorphous GaAs. ${ }^{14,15,18}$ These results indicate that the GaAs film crystallizes at $T_{\mathrm{g}} \geq 370^{\circ} \mathrm{C}$. Figure 2(a) also shows that the $\mathrm{GaAs}$ films grown on c-Ge and bare glass substrates are crystalline at $T_{\mathrm{g}}=520^{\circ} \mathrm{C}$. The Raman peaks of the samples with ALILE-Ge are almost as sharp as those of the c-Ge sample, while those of the glass sample are relatively broad. These results indicate that the GaAs film on ALILE-Ge has high crystallinity. Compared to the c-Ge sample, the samples with ALILE-Ge and the glass sample have the GaAs peaks slightly shifted to the lower wavenumber. This is likely due to the stress derived from the difference in the thermal expansion coefficient between GaAs and glass. The EBSD images in Figs. 2(b) and 2(f) show that the $T_{\mathrm{g}}=420^{\circ} \mathrm{C}$ sample with ALILE-Ge has lower (111) orientation and a smaller grain size than those of the ALILE-Ge layer [Figs. 1 (b) and 1(c)], indicating the incomplete epitaxial growth of GaAs. Conversely, Figs. 2(c) and 2(g) show that the (111) orientation fraction and the grain size of the $T_{\mathrm{g}}=520^{\circ} \mathrm{C}$ sample with ALILE-Ge are the same as the ALILE-Ge layer [Figs. 1(b) and 1(c)]. These results suggest that the GaAs film was epitaxially grown from the ALILE-Ge seed layer at $T_{\mathrm{g}}=520^{\circ} \mathrm{C}$. The epitaxial growth of GaAs at $T_{\mathrm{g}}=520^{\circ} \mathrm{C}$ is also confirmed for the c-Ge sample from Figs. 2(d) and 2(h). Figures 2(e) and 2(i) show that the GaAs film directly grown on glass has very small grains $(<100 \mathrm{~nm})$ which are below the detection limit of the EBSD system. Thus, the pseudosingle crystal GaAs layer (grain size $>100 \mu \mathrm{m}$ ) was achieved below the heat proof temperature of general soda-lime glass $\left(\sim 560^{\circ} \mathrm{C}\right)$.

After preparing ITO electrodes, we evaluated the detailed crosssectional structure of the sample with ALILE-Ge for $T_{\mathrm{g}}=520^{\circ} \mathrm{C}$ using a transmission electron microscope (TEM; FEI Tecnai Osiris) operating at $200 \mathrm{kV}$, equipped with an energy-dispersive X-ray spectrometer (EDX). Figures 3(a) and 3(b) show the stacked structure of ITO/GaAs/ $\mathrm{Ge} /$ glass as intended. The surface of the GaAs film is flatter than that of the ALILE-Ge layer likely due to the appearance of a (111) facet during MBE. The selected-area electron diffraction (SAED) pattern in Fig. 3(c) indicates that the GaAs film is epitaxially (111) oriented and single crystalline in this area (800 $\mathrm{nm}$ in diameter). Figures $3(\mathrm{~d})$ and 3(e) show that the GaAs film contains dislocations and stacking faults, whereas the Ge seed layer is free from extended defects. ${ }^{28}$ Such defects, mostly extended from the epitaxial interface, were also found in the Ge film homoepitaxially grown on ALILE-Ge at $500^{\circ} \mathrm{C} .^{32}$ Figures $3(\mathrm{f})-3(\mathrm{~h})$ show that (111) planes are in an orderly line up from the $\mathrm{Ge} /$ glass interface to the GaAs film. The GaAs/Ge interface is so continuous that it is difficult to identify from the lattice image [Fig. $3(\mathrm{~g})$ ]. The cause of the defects in GaAs is presumed to be the rough surface of the ALILE-Ge layer, the low growth temperature of GaAs, and/or 
(a)

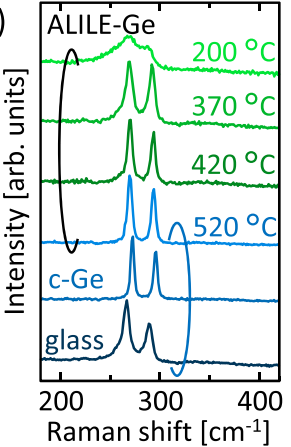

(b) $1920 \cdot c$
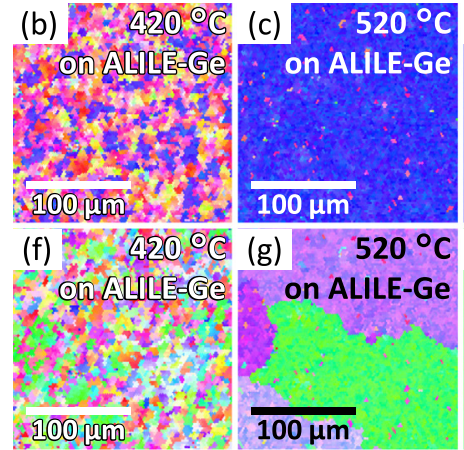

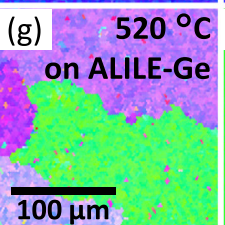

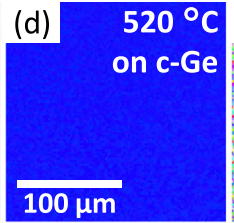

(h)

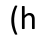

$520^{\circ} \mathrm{C}$ on $\mathrm{C}-\mathrm{Ge}$

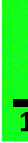

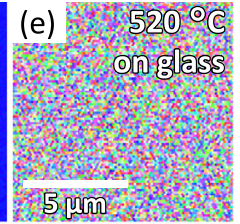

(i) 5200 the difference in the thermal expansion coefficients between GaAs and the $\mathrm{SiO}_{2}$ glass substrate. To form a thick GaAs film, it seems important to select a specific glass substrate in consideration of the thermal expansion coefficient. To evaluate the minority carrier lifetime of GaAs directly related to solar cell performance, microwave
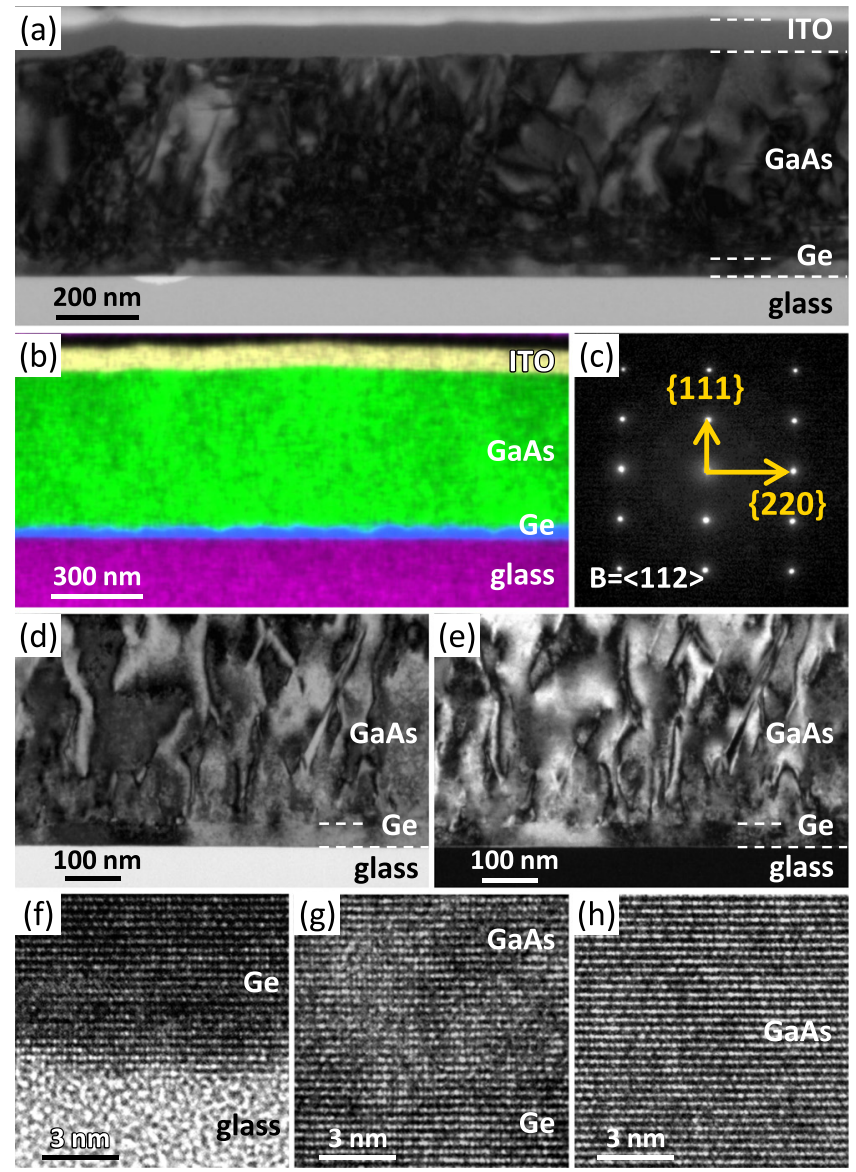

FIG. 3. Cross-sectional TEM characterization of the sample with ALILE-Ge for $T_{\mathrm{g}}$ $=520^{\circ} \mathrm{C}$. (a) Low-magnification bright-field TEM image. (b) EDX elemental mapping. (c) SAED pattern taken from a region including all layers with a selected area of diameter $800 \mathrm{~nm}$. (d) High-magnification bright-field TEM image. (e) Dark-field TEM image using the (111) plane reflection. High-resolution lattice images showing the (f) Ge/glass interface, (g) GaAs/Ge interface, and (h) GaAs film. photoconductivity decay measurements (excitation wavelength $349 \mathrm{~nm}$ microwave frequency $26 \mathrm{GHz}$ ) were performed. The results indicated that the minority carrier lifetimes of the GaAs layers on both ALILE-Ge and c-Ge were lower than the time resolution (5ns) of the microwave photoconductivity decay system. To improve the minority carrier lifetime, further optimization of the growth conditions of GaAs is necessary.

Photoresponsivity was measured for the sample with ALILE-Ge for $T_{\mathrm{g}}=520^{\circ} \mathrm{C}$ using the structure illustrated in Fig. 4(a). The resistivity of ALILE-Ge is low enough $\left(<10^{-3} \Omega \mathrm{cm}\right)$ to be used as a bottom electrode due to highly $\mathrm{Al}$ doping. ${ }^{35}$ The bias voltages were applied to (a)
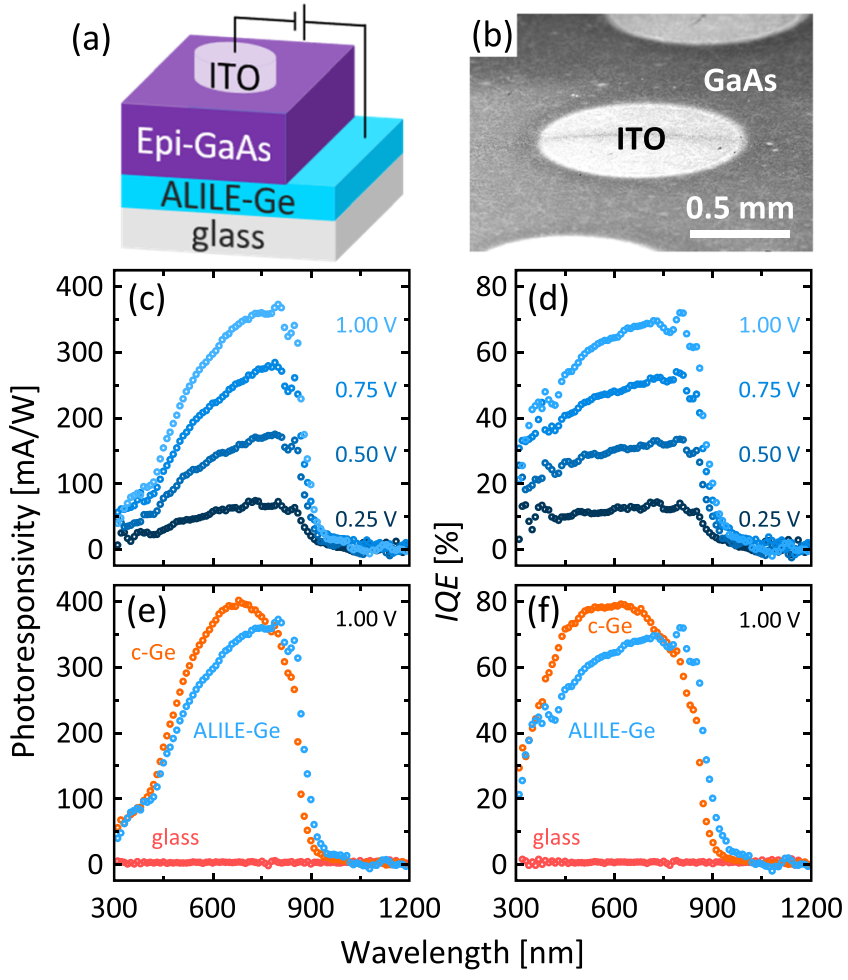

FIG. 4. Photoresponse properties of the samples with $T_{g}=520^{\circ} \mathrm{C}$. (a) Schematic of the sample for the measurement. (b) SEM image of ITO electrodes patterned on the GaAs film grown on ALILE-Ge. (c) Bias voltage-dependent photoresponsivity and (d) IQE of the sample with ALILE-Ge. (e) Photoresponsivity and (f) IQE of the samples with ALILE-Ge, c-Ge, and glass where the bias voltage is $1.0 \mathrm{~V}$. 
the ALILE-Ge layer with respect to the ITO electrode, prepared as shown in the scanning electron microscopy (SEM) image in Fig. 4(b). Because the current sample does not have a PN junction in GaAs, the photogenerated holes in GaAs are transferred to the surface ITO electrode via drift. Figure 4(c) shows clear photoresponse spectra rising near a wavelength of $900 \mathrm{~nm}$ corresponding to the GaAs bandgap with each bias voltage. Figure 4 (d) shows that internal quantum efficiency (IQE) reaches $70 \%$ with a bias voltage of $1.0 \mathrm{~V}$. Figures $4(\mathrm{e})$ and 4(f) show that the sample with ALILE-Ge exhibits photoresponsivity which is comparable to that of the c-Ge sample, while the glass sample exhibits no photoresponsivity. The high photoresponsivity of the sample with ALILE-Ge is owing to the GaAs film being pseudosingle crystal. Compared to the c-Ge sample, the sample with ALILE-Ge exhibits the rising edge of the photoresponsivity slightly shifted to the longer wavelength, suggesting that the bandgap of GaAs becomes pseudonarrower due to the shallow level defects. In addition, the photoresponsivity of the sample with ALILE-Ge begins to decrease at the short wavelength side $(<850 \mathrm{~nm})$ likely because of the defects in the region close to the surface of the GaAs film. Therefore, further investigation of low temperature growth of GaAs will be able to improve the photoresponsivity, which is the largest currently for the GaAs film grown on a glass substrate.

In conclusion, we demonstrated the great potential of ALILE-Ge as a seed layer for GaAs thin-film solar cells. The GaAs film grown epitaxially from ALILE-Ge on glass at $520^{\circ} \mathrm{C}$ became a pseudosingle crystal (grain size $>100 \mu \mathrm{m}$ ) with high (111) orientation. Reflecting the large grain size, the $I Q E$ reached $70 \%$ under a bias voltage of $1.0 \mathrm{~V}$. This value approached the simultaneously formed GaAs film on c-Ge and is the highest value for a GaAs film synthesized on glass. The achievements will open up the possibility of developing high-efficiency thin-film solar cells with III-V compound semiconductors based on low-cost glass substrates.

This work was supported financially by the JSPS KAKENHI (No. 17H04918). The authors are grateful to Dr. Y. Tominaga (Hiroshima University) for helpful discussions and Professor N. Usami (Nagoya University) for assistance with the microwave photoconductivity decay measurement. Some experiments were conducted at the International Center for Young Scientists at NIMS and the Nanotechnology Platform at the University of Tsukuba.

\section{REFERENCES}

${ }^{1}$ M. A. Green, Y. Hishikawa, E. D. Dunlop, D. H. Levi, J. Hohl-Ebinger, and A. W. Y. Ho-Baillie, Prog. Photovoltaics: Res. Appl. 26, 427 (2018).

${ }^{2}$ M. Yamaguchi, T. Takamoto, K. Araki, and N. Ekins-Daukes, Sol. Energy 79, 78 (2005).

${ }^{3}$ R. R. King, D. C. Law, K. M. Edmondson, C. M. Fetzer, G. S. Kinsey, H. Yoon, R. A. Sherif, and N. H. Karam, Appl. Phys. Lett. 90, 183516 (2007).

${ }^{4}$ R. Venkatasubramanian, E. Siivola, B. O'Quinn, B. Keyes, and R. Ahrenkiel, AIP Conf. Proc. 411, 411 (1997).

${ }^{5}$ J. Yoon, S. Jo, I. S. Chun, I. Jung, H.-S. Kim, M. Meitl, E. Menard, X. Li, J. J. Coleman, U. Paik, and J. A. Rogers, Nature 465, 329 (2010).
${ }^{6}$ D. Shahrjerdi, S. W. Bedell, C. Ebert, C. Bayram, B. Hekmatshoar, K. Fogel, P. Lauro, M. Gaynes, T. Gokmen, J. A. Ott, and D. K. Sadana, Appl. Phys. Lett. 100, 053901 (2012).

${ }^{7}$ E. U. Onyegam, D. Sarkar, M. Hilali, S. Saha, R. A. Rao, L. Mathew, D. Jawarani, J. Mantey, M. Ainom, R. Garcia, W. James, and S. K. Banerjee, Sol. Energy Mater. Sol. Cells 111, 206 (2013).

${ }^{8}$ S. Moon, K. Kim, Y. Kim, J. Heo, and J. Lee, Sci. Rep. 6, 30107 (2016).

${ }^{9}$ M. Yamaguchi and Y. Itoh, J. Appl. Phys. 60, 413 (1986).

${ }^{10}$ S. R. Kurtz and R. McConnell, AIP Conf. Proc. 404, 191 (1997).

"1. J. J. Yang, P. D. Dapkus, R. D. Dupuis, and R. D. Yingling, J. Appl. Phys. 51, 3794 (1980).

${ }^{12}$ S. Tsuji, E. Iri, and H. Takakura, Jpn. J. Appl. Phys., Part 1 31, 880 (1992).

${ }^{13}$ K. Mochizuki, T. Nakamura, T. Mishima, H. Masuda, and T. Tanoue, J. Electron. Mater. 23, 577 (1994).

${ }^{14}$ M. Imaizumi, M. Adachi, Y. Fujii, Y. Hayashi, T. Soga, T. Jimbo, and M. Umeno, J. Cryst. Growth 221, 688 (2000).

${ }^{15}$ A. Erlacher, B. Ullrich, E. Y. Komarova, H. Jaeger, H. J. Haugan, and G. J. Brown, J. Non Cryst. Solids 352, 193 (2006).

${ }^{16}$ Y. Kajikawa, T. Okuzako, S. Takami, and M. Takushima, Thin Solid Films 519, 136 (2010).

${ }^{17}$ V. Şenay, S. Özen, S. Pat, and Ş. Korkmaz, J. Alloys Compd. 663, 829 (2016).

${ }^{18}$ R. Campomanes, J. Dias da Silva, J. Vilcarromero, and L. Cardoso, J. Non Cryst. Solids 299-302, 788 (2002).

${ }^{19}$ J. H. Epple, K. L. Chang, C. F. Xu, G. W. Pickrell, K. Y. Cheng, and K. C. Hsieh, J. Appl. Phys. 93, 5331 (2003).

${ }^{20}$ D. Pirzada and G. J. Cheng, J. Appl. Phys. 105, 093114 (2009).

${ }^{21}$ J. Nayak and S. N. Sahu, Appl. Surf. Sci. 182, 407 (2001).

${ }^{22}$ S. Sathasivam, R. R. Arnepalli, B. Kumar, K. K. Singh, R. J. Visser, C. S. Blackman, and C. J. Carmalt, Chem. Mater. 26, 4419 (2014).

${ }^{23}$ M. G. Mauk, J. R. Balliet, and B. W. Feyock, J. Cryst. Growth 250, 50 (2003).

${ }^{24}$ C.-Y. Tsao, J. Huang, X. Hao, P. Campbell, and M. A. Green, Sol. Energy Mater. Sol. Cells 95, 981 (2011).

${ }^{25}$ P. Dutta, M. Rathi, D. Khatiwada, S. Sun, Y. Yao, B. Yu, S. Reed, M. Kacharia, J. Martinez, A. P. Litvinchuk, Z. Pasala, S. Pouladi, B. Eslami, J.-H. Ryou, H. Ghasemi, P. Ahrenkiel, S. Hubbard, and V. Selvamanickam, Energy Environ. Sci. 12, 756 (2019).

${ }^{26}$ Z. M. Wang, J. Y. Wang, L. P. H. Jeurgens, F. Phillipp, and E. J. Mittemeijer, Acta Mater. 56, 5047 (2008).

${ }^{27}$ S. Hu, A. F. Marshall, and P. C. McIntyre, Appl. Phys. Lett. 97, 082104 (2010).

${ }^{28}$ K. Toko, M. Kurosawa, N. Saitoh, N. Yoshizawa, N. Usami, M. Miyao, and T. Suemasu, Appl. Phys. Lett. 101, 072106 (2012).

${ }^{29}$ J.-H. Park, T. Suzuki, M. Kurosawa, M. Miyao, and T. Sadoh, Appl. Phys. Lett. 103, 082102 (2013)

${ }^{30}$ K. Toko, R. Numata, N. Oya, N. Fukata, N. Usami, and T. Suemasu, Appl. Phys. Lett. 104, 022106 (2014).

${ }^{31}$ K. Toko, K. Nakazawa, N. Saitoh, N. Yoshizawa, N. Usami, and T. Suemasu, CrystEngComm 16, 2578 (2014).

${ }^{32}$ K. Toko, K. Nakazawa, N. Saitoh, N. Yoshizawa, and T. Suemasu, Cryst. Growth Des. 15, 1535 (2015).

${ }^{33}$ N. Oya, K. Toko, N. Saitoh, N. Yoshizawa, and T. Suemasu, Appl. Phys. Lett. 104, 262107 (2014).

${ }^{34}$ H. Higashi, K. Kasahara, K. Kudo, H. Okamoto, K. Moto, J.-H. Park, S. Yamada, T. Kanashima, M. Miyao, I. Tsunoda, and K. Hamaya, Appl. Phys. Lett. 106, 041902 (2015).

${ }^{35}$ K. Kusano, A. Yamamoto, M. Nakata, T. Suemasu, and K. Toko, ACS Appl. Energy Mater. 1, 5280 (2018).

${ }^{36}$ T. Kawai, H. Yonezu, H. Yoshida, and K. Pak, Appl. Phys. Lett. 61, 1216 (1992). 